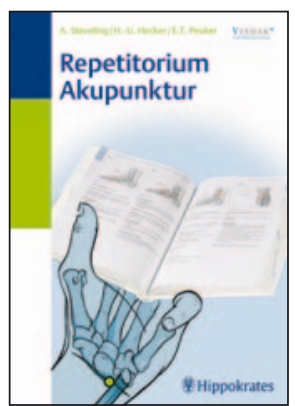

Steveling, A.; Hecker, H.-U.;

Peuker, E.T.

\section{Repetitorium Akupunktur}

Stuttgart, Hippokrates, 2009, 327 S., 49,95 EUR/

$82,90 \mathrm{CHF}$

ISBN 978-3-8304-5412-0

Klinische Nützlichkeit: Dieses neue Buch ist sehr übersichtlich gestaltet, enthält viele Tabellen und ist in verschiedenen Farben gedruckt. Die Gliederung in «Theorieteil» und «Praxisteil» erweist sich als nützlich. So enthält der Theorieteil die Grundlagen der Akupunktur sowie die Durchführung der Akupunkturbehandlung. Dieser Buchteil ist ausführlich unter anderem mit Indikationen und Kontraindikationen, Patientenlagerung und Hinweisen zu Akupunkturnadeln (Material, Stärke, Länge und Wiederverwertbarkeit), aber auch mit Hinweisen zu Akupressur, TENS, Schröpfen sowie zur Moxibustion versehen. Der Praxisteil umfasst Erkrankungen des Bewegungsapparates, der inneren Medizin und gynäkologische Krankheiten, die in Bild und Text ziemlich umfassend dargestellt werden.

Offenbar ist dieses Lehrbuch für die Prüfungen zur «Zusatzbezeichnung Akupunktur» in Deutschland als Grundlagenwerk geeignet. Da die Autoren in unterschiedlichen Akupunkturgesellschaften und Landesärztekam- mern tätig sind, haben sie verschiedene Betrachtungsansätze, die jedoch alle die kurrikularen Anforderungen der Bundesärztekammer erfüllen. Für die Ausbildungsmodule in der Schweiz, die zu einem Fähigkeitsausweis TCM - Akupunktur der Foederatio Medicorum Helveticae führen, ist das Buch mit folgender Einschränkung empfehlenswert: Es fehlen die englischen und chinesischen Punktebezeichnungen. Dieses Manko ist recht schwerwiegend, denn einerseits hat sich international die englische Schreibweise durchgesetzt, so beispielsweise ST36 anstelle von Ma 36. Andererseits ist für Lernaufenthalte in China die Kenntnis der chinesischen Namen der Akupunkturpunkte unabdingbar.

Originalität: Gut gemacht sind die jeweils am Ende eines Kapitels eingefügten Rubriken mit Fragen und Antworten zum Selbststudium, simulieren sie doch die mündliche Prüfung in lerntechnisch gut aufbereiteter Form. Erfreulich ist auch die Übersicht zur Ohrakupunktur, weil sie die westliche - und hier die französische und die russische Schule - und die chinesische Sichtweise darstellt. Im Praxisteil wurde viel Sorgfalt auf ein strukturiertes Diagnoseund Therapiekonzept verwendet, das in vier Schritte gegliedert ist.

Wissenschaftliche Nützlichkeit: Zwei Seiten sind den deutschen Art- und Gerac-Studien gewidmet; dies mit genauen Literaturangaben. Eine Option für eine Überarbeitung des Buches wären Therapiebeispiele auf der Basis von wissenschaftlichen Forschungsergebnissen. Die streng wissenschaftliche Nützlichkeit dieses Buches hält sich in Grenzen. Ohne wissenschaftliche Grundlagen aber, die sich auf klinische Studien beziehen, wird die Akupunktur im
Westen dereinst einen schweren Stand haben. Es empfiehlt sich daher, schon die Studienanfänger für dieses Thema zu sensibilisieren.

Präsentation: Zunächst habe ich mich gefragt, ob es wirklich notwendig und sinnvoll ist, dass schon wieder ein neues Akupunkturbuch auf den Markt kommt. Mein erster Eindruck war bei diesem 327 Seiten starken Buch eher negativ, da das Titelbild eine zum Teil nur aus Knochen bestehende Hand zeigt, die das aufgeschlagene Buch auf den Seiten 91 und 92 hält. Nun ja, zum Skelett und später zu Staub werden wir alle dereinst fortschreiten, aber ist das die richtige Assoziation für die Hand eines Lernenden? Oder sind Ärzte tatsächlich so abgebrüht, dass sie sich an einem solchen Bild nicht stossen?

Beim Blättern im Buch sieht man, dass alle Abbildungen auf anatomische Gegebenheiten bezogen und so für Mediziner gut verständlich sind. Das Papier ist griffig und strapazierfähig, es lädt zu häufigem Nachschlagen ein, der Umschlag ist abwaschbar, was sich als nützlich erweisen wird!

Der Lernende gewinnt eine gute Übersicht durch Fettgedrucktes oder mit blauen Punkten versehene Auflistungen; diese gewährleisten eine schnelle Orientierung vor allem für visuell begabte Lernende. Ein schön aufgebautes Literaturverzeichnis, das nicht nur Bücher, sondern auch Studien erwähnt, und ein übersichtliches Sachverzeichnis runden das Werk ab.

Wer sich selbst ein Bild über dieses Buch machen möchte, kann das im gelungenen Internetauftritt unter www.medizinverlage.de/detailseiten/ 9783830454120.html tun.

Brigitte Ausfeld-Hafter, Bern

\section{KARGER}




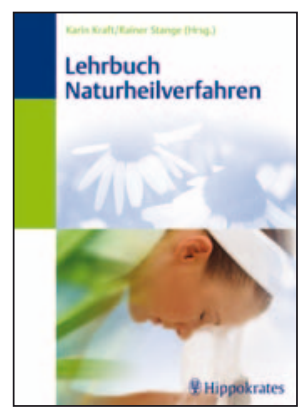

Kraft, K.; Stange, R. (Hrsg)

Lehrbuch Naturheilverfahren

Stuttgart, Hippokrates, 2009, 839 S., 99,95 EUR/ $166,00 \mathrm{CHF}$

ISBN 978-3-8304-5333-8

Kürzlich erschienen ist das von Karin Kraft und Rainer Stange herausgegebene «Lehrbuch Naturheilverfahren», das sich als Nachfolger des Standardwerks der 1980/90er-Jahre, dem «Schimmel», einordnet und nun nach jahrelanger Lücke in einem sehr umfangreichen Band (839 Seiten) vorliegt. Dieses Buch widmet sich dem Thema Naturheilkunde in umfassender Weise - andere Verfahren der Komplementärmedizin werden lediglich in abschliessenden Kapiteln auf jeweils wenigen Seiten kurz angerissen. Bei einem solchen Buch mit Beiträgen von 44 Autoren nimmt man gerne in Kauf, dass sich die einzelnen Kapitel je nach Autor deutlich im Stil unterscheiden. Ein Lehrbuch verzichtet auf detaillierte Zitate und beschränkt sich meist auf allgemeine und weiterführende Hinweise $\mathrm{zu}$ wichtiger Literatur und eventuell einige Schlüsselstudien zum jeweiligen Kapitel. Das Buch mit einem Geleitwort von Prof. Reinhard Saller wendet sich an Anfänger und bietet dabei auch Fortgeschrittenen in der Naturheilkunde eine Fundgrube an Hinweisen, Anregungen und Ideen.

Im einleitenden Kapitel werden Besonderheiten und Ansprüche der Naturheilkunde dargelegt. Allerdings findet man dort keine Erklärung der ungewöhnlichen Einordnung von Elektrotherapie und Ultraschall als «klassische Naturheilverfahren» im Buch. In Deutschland gibt es aber eine offizielle ärztliche Weiterbildung zur Zusatzbezeichnung "Naturheilverfahren», bei der diese Gebiete eingeschlossen sind - nicht aufgrund sachlicher Aspekte, sondern wohl eher aufgrund früherer berufspolitischer Aktivitäten des ehemals starken Fachgebiets Physikalische Medizin, zu dem ja etliche Überlappungen bestehen.

Allgemeine Kapitel über Naturheilverfahren in Prävention, Rehabilitation sowie ärztlicher Praxis stecken die Bandbreite der Naturheilkunde ab, lassen jedoch naturheilkundliche Besonderheiten oder Ansprüche in diesen Gebieten kaum erkennen. Hier werden jeweils bereits Beispiele von naturheilkundlichen Behandlungsansätzen dargestellt.

Zum Selbstverständnis der Naturheilkunde trägt der übersichtliche und spannend zu lesende geschichtliche Abriss bei. Es folgen die Kapitel über die wichtigsten Verfahren der Naturheilkunde, beginnend mit Diagnoseverfahren, Ordnungstherapie und dann Chronobiologie. Das Kapitel über Ordnungstherapie wirkt etwas knapp. Bei der Phytotherapie werden nach kurzer Einführung in die Grundsätze wichtige Pflanzen monographieartig dargestellt - wobei unklar bleibt, woher die Indikationsangaben stammen. Neuere praxisrelevante Erkenntnisse wird man hier nur gelegentlich finden, ebenso wenig wie Beispiele zu Rezepturen oder zu bekannten Präparaten. Bei der Hydrotherapie erwecken allzu spezifische Indikationszuordnungen der detailliert dargestellten einzelnen Wasseranwendungen eine falsche Sichtweise über die doch vorwiegend unspezifischen Möglichkeiten einer kurmässig oder ständig durchgeführten Hydrotherapie. Die vielen Details können einen Anfänger eher verwirren und abschrecken, sich näher auf die Hydrotherapie einzulassen. Einen roten Faden lassen dieselben Autoren besser bei dem nachfolgenden Kapitel über Sauna, Dampfbad und Ganzkörperhyperthermie erkennen. Das Kapitel über Bewegungstherapie nennt vermehrte körperliche Aktivität, Sporttherapie und Krankengymnastik, gibt aber nur bei einzelnen Krankheitsbildern konkrete Angaben zur Intensität, Dauer und Häufigkeit entsprechender Aktivitäten. Es folgt ein gut verständliches und bebildertes Kapitel über Manuelle Medizin. Ein naturheilkundlicher Bezug ist bei dem ernährungsphysiologisch ausgerichteten Kapitel zur Ernährung kaum erkennbar, während spezielle naturheilkundliche Sichtweisen bei den Kapiteln über Heilfasten bzw. Mayr-Therapie deutlich werden. Das Kapitel Atem- und Entspannungstherapien geht recht knapp auf die wichtigsten Verfahren ein; es folgen ausführlicher erscheinende Kapitel über Klimatherapie, Heliotherapie, sowie dann (!) Ultraschall- und Elektrotherapie. Als erweiterte Verfahren folgen Kapitel über Akupunktur, Neuraltherapie, Ausleitende Verfahren, Eigenblut, Sauerstoff-Ozontherapie, Mikrobiologische Therapie sowie Segment- und Reflexzonenbehandlung.

Danach folgen die fachspezifischen Kapitel, die eine Fülle von praktischen Hinweisen auch für fortgeschrittene Naturheilärzte bieten. Die Reihenfolge der einzelnen Verfahren bei der jeweiligen Indikation variiert je nach ihrer Bedeutung für die jeweilige Indikation, und mitunter fallen dann die vom jeweiligen Autor als weniger wichtig angesehenen Verfahren auch ganz weg. Aus dem Rahmen fällt allerdings das durchgängige Erscheinen homöopathischer Empfehlungen im Kapitel Gynäkologie.

Anhand von deutlich markierten Seiten lässt sich immer schnell feststellen, in welchem Teil des Buches man sich gerade befindet.

Anstelle des mehrseitigen Abbildungsnachweises wäre ein etwas ausführlicherer Index wünschenswert; die Nachschlagemöglichkeiten sind brauchbar, könnten aber noch ausführlicher und kompletter sein.

Letztendlich sprechen aber diese kleinen Kritikpunkte nicht dagegen, dass dieses Buch sich als Standardwerk der Naturheilkunde entwickeln wird. Es hat einfach das Potenzial, ein Klassiker zu werden, und kann hoffentlich in weiteren Auflagen noch abgerundet und an neueste Entwicklungen angepasst werden.

Bernhard Uehleke, Berlin 